\title{
Elucidating Mass Transport Regimes in Gas Diffusion Electrodes for $\mathrm{CO}_{2}$ Electroreduction
}

\author{
Electronic Supporting Information
}

Thomas Moore*, Xiaoxing Xia, Sarah E. Baker, Eric B. Duoss and Victor A. Beck*

Lawrence Livermore National Laboratory, 7000 East Ave., Livermore, CA, USA, 94551

Email:moore260@llnl.gov; beck33@llnl.gov

\section{Section S1. Supplementary Figures}

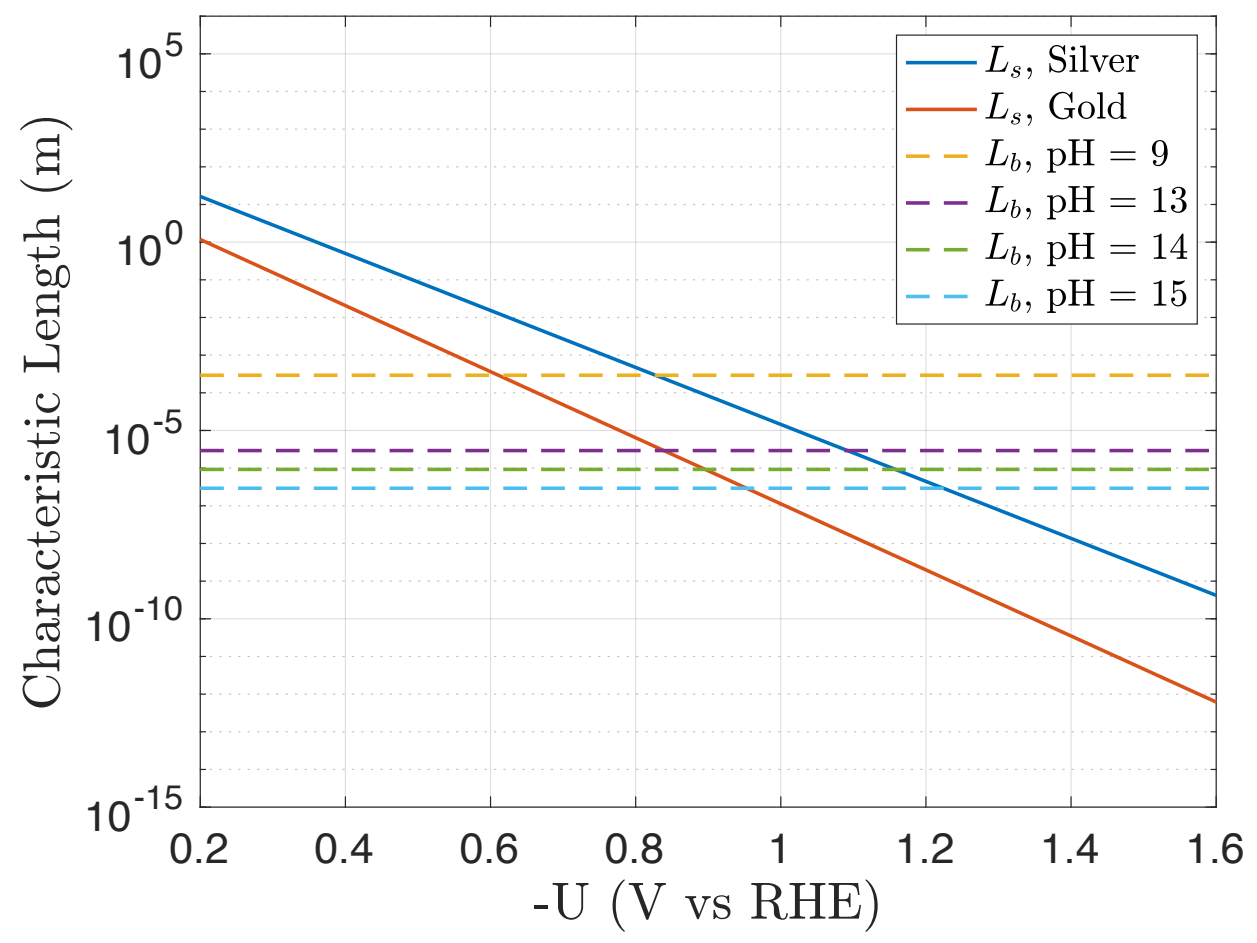

Figure S1: Characteristic length-scales for bulk reaction control $\left(L_{b}\right)$ and Triple Phase Region control $\left(L_{s}\right)$ as a function of $\mathrm{pH}$ and applied potential,for COER on Ag and Au catalysts.

Parameters sourced from Table 1. 


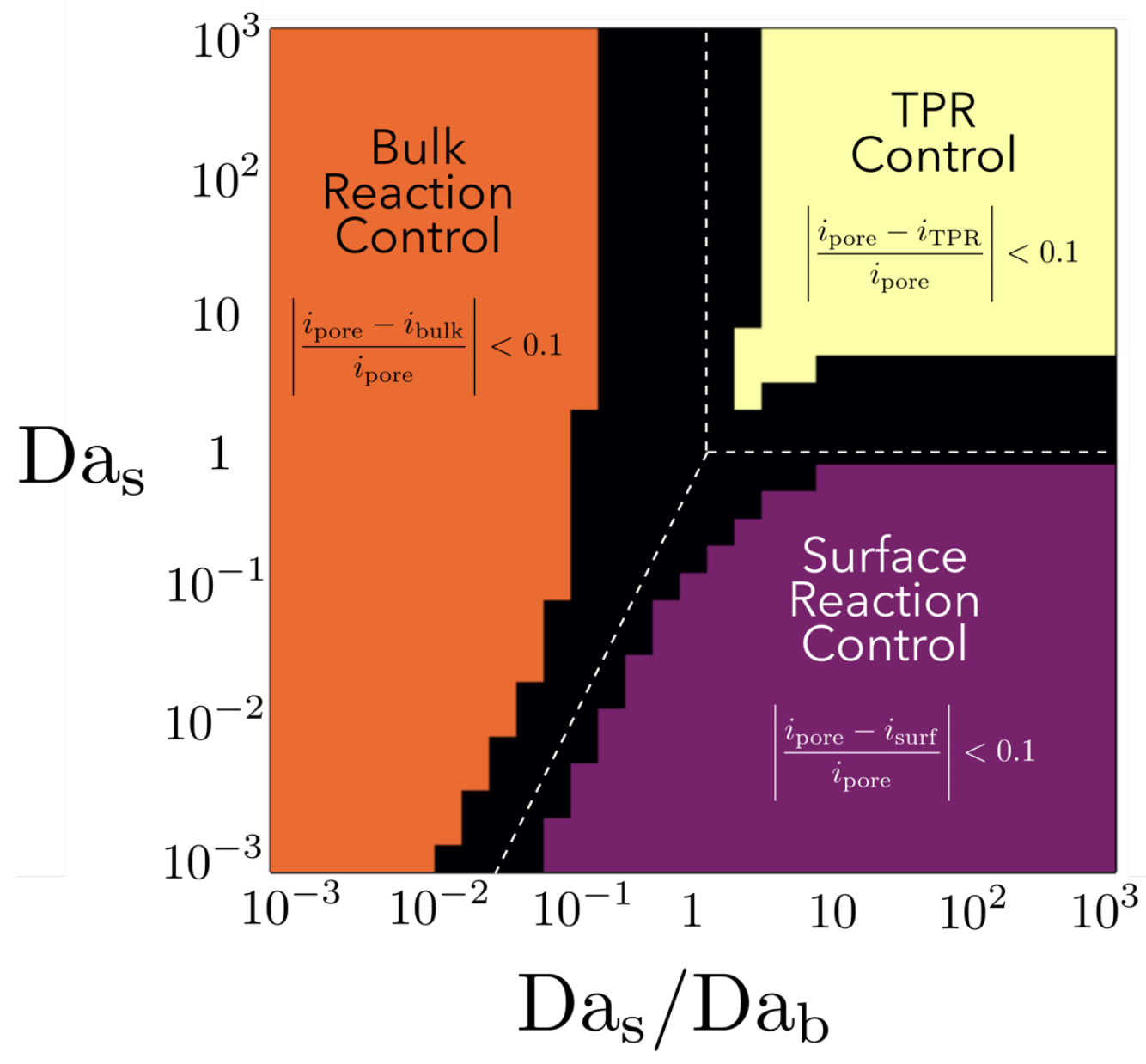

Figure S2: Numerically generated phase diagrams, showing regions in which the various asymptotic approximations for the $\mathrm{CO}_{2} \mathrm{R}$ current density listed in Table 2 of the main paper are within $10 \%$ of the resolved $\mathrm{CO}_{2}$ pore model. Black regions are transitional regions in which no asymptotic approximations are correct within 10\%. Dashed lines show theoretical phase boundaries from Figure 3 in main paper. 


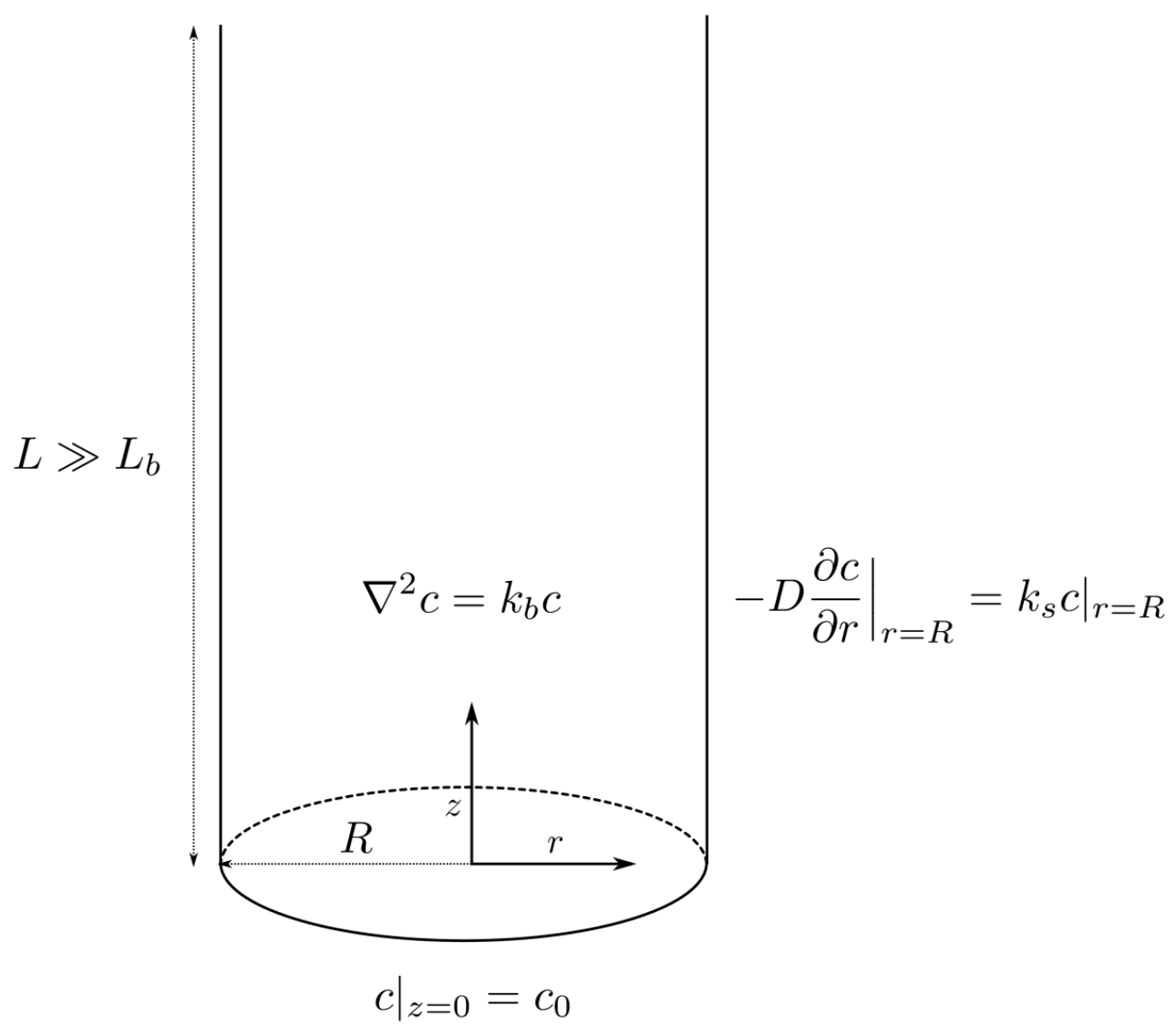

Figure S3

Figure S3: Geometry, PDEs and boundary conditions for resolved $\mathrm{CO}_{2}$ pore model, in which it is it assumed that the $\mathrm{pH}$ and electric potential are uniform within each pore. Pore length is fixed to be much larger than $\mathrm{L}_{b}$, ensuring finite-length effects do not influence $\mathrm{CO}_{2}$ transport. 


\section{Section S2. Development of Multiphysics Model.}

A multiphysics model of the diffusion-reaction-migration processes occurring in the vicinity of a triple phase boundary was developed using the commercial software StarCCM.

Governing Equations

Diffusion-migration and reaction of $\mathrm{CO}_{2}, \mathrm{CO}_{3}{ }^{2}-, \mathrm{OH}^{-}, \mathrm{K}^{+}, \mathrm{H}_{2}$ and $\mathrm{CO}$ were modelled via the Nernst-Planck equation:

$$
\frac{\partial \mathrm{c}_{\mathrm{i}}}{\partial \mathrm{t}}=\nabla \cdot\left(\mathrm{D}_{\mathrm{i}} \nabla \mathrm{c}_{\mathrm{i}}+\frac{\mathrm{z}_{\mathrm{i}} \mathrm{F}}{\mathrm{RT}} \mathrm{c}_{\mathrm{i}} \nabla \phi\right)+r_{i}
$$

where $c_{i}$ is the concentration of species $i, t$ is the time, $D_{i}$ is the diffusivity of $i, z_{i}$ is the charge of $\mathrm{i}, \mathrm{F}$ is Faraday's constant, $R$ is the ideal gas constant, $T$ is the temperature, $\phi$ is the potential, and $r_{i}$ is the rate of production of $i$ per unit volume. Diffusion coefficients for each species are provided in Table 1 of the main paper; electrical mobilities were calculated via the NernstEinstein equation. The electric potential distribution was determined by the condition of charge neutrality, which was imposed everywhere within the liquid electrolyte domain. Convection was ignored, as the local Peclet number is negligible in the vicinity of the solid surface.

$\mathrm{CO}_{2}$ and $\mathrm{OH}^{-}$reacted according to the following reaction, which may be considered irreversible in basic $(\mathrm{pH}>12)$ solutions:

$$
\mathrm{CO}_{2}+2 \mathrm{OH}^{-} \rightarrow \mathrm{CO}_{3}^{2-}+\mathrm{H}_{2} \mathrm{O}
$$

The overall reaction stoichiometry is a result of two reactions in series: the slow, rate-limiting reaction of $\mathrm{CO}_{2}$ with $\mathrm{OH}^{-}$:

$$
\mathrm{CO}_{2}+\mathrm{OH}^{-} \rightarrow \mathrm{HCO}_{3}^{-}
$$

and the instantaneous proton-transfer:

$$
\mathrm{HCO}_{3}^{-}+\mathrm{OH}^{-} \rightarrow \mathrm{CO}_{3}^{2-}+\mathrm{H}_{2} \mathrm{O}
$$

both reactions may be considered irreversible in solutions with $\mathrm{pH}>12$; see Danckwerts (1970, p. 240) and Fukunaka (1989).

The reaction of $\mathrm{CO}_{2}$ with $\mathrm{OH}^{-}$is rate controlling, leading to a second order reaction rate of the form:

$$
-r_{\mathrm{CO}_{2}}=k_{2} c_{\mathrm{CO}_{2}} c_{\mathrm{OH}^{-}}
$$

\section{Geometry and Boundary Conditions}

Diffusion-reaction-migration equations were simulated within an electrolyte contained in a pore of varying radius and with length $10 \mu \mathrm{m}$ (see Figure 2 in the paper). At the bottom of the pore 
was a gas-liquid interface, at which the concentration of dissolved gases $\left(\mathrm{CO}_{2}, \mathrm{CO}\right.$ and $\left.\mathrm{H}_{2}\right)$ were specified via Dirichlet conditions:

$$
\mathrm{c}_{\mathrm{CO}_{2}}^{\text {Gas-Liq Interface }}=\mathrm{c}_{\mathrm{CO}_{2}}^{*} ; \quad \mathrm{c}_{\mathrm{CO}}^{\text {Gas-Liq Interface }}=\mathrm{c}_{\mathrm{CO}}^{*} ; \quad \mathrm{c}_{\mathrm{H}_{2}}^{\text {Gas-Liq Interface }}=\mathrm{c}_{\mathrm{H}_{2}}^{*} ;
$$

$c_{\mathrm{CO}_{2}}^{*}$ was set to $0.015 \mathrm{M}$, while $c_{C O}^{*}$ and $c_{\mathrm{H}_{2}}^{*}$ was set to $0 \mathrm{M}$. Homogeneous Neumann conditions were used for all other species. At the top of the pore, the concentration of dissolved species were set to the following values:

$$
\begin{aligned}
& c_{\mathrm{CO}_{2}}^{\text {Top of Pore }}=0 \mathrm{M} \\
& c_{\mathrm{CO} \text { of Pore }}^{\text {Top }}=0 \mathrm{M} \\
& c_{\mathrm{OH}^{-}}^{\text {Top Pore }}=1 \mathrm{M} \\
& c_{\mathrm{K}^{+}}^{\text {Top of Pore }}=1 \mathrm{M} \\
& \mathrm{c}_{\mathrm{CO}_{3}^{2-}}^{\text {Top of Pore }}=0 \mathrm{M}
\end{aligned}
$$

The electrical potential at the top of the pore was set to $0 \mathrm{~V}$.

The electrical potential of the cathode was varied, with different values corresponding to different values of $\lambda$. Both COER and HER reactions were modelled at the cathode surface:

$$
\begin{gathered}
\mathrm{CO}_{2}+\mathrm{H}_{2} \mathrm{O}+2 \mathrm{e}^{-} \rightarrow \mathrm{CO}+2 \mathrm{OH}^{-} \\
2 \mathrm{H}_{2} \mathrm{O}+2 \mathrm{e}^{-} \rightarrow \mathrm{H}_{2}+2 \mathrm{OH}^{-}
\end{gathered}
$$

The former was modelled via a mass-transfer limited Tafel expression,

$$
i=i_{C O E R}^{0} \frac{c_{C O_{2}}}{c_{C O_{2}}^{0}} \exp \left(-\frac{\alpha_{C O E R} F}{R T}\left(V-U_{C O E R}^{0}\right)\right)
$$

While the latter was modelled via a standard Tafel expression,

$$
i=i_{H E R}^{0} \exp \left(-\frac{\alpha_{H E R} F}{R T}\left(V-U_{H E R}^{0}\right)\right)
$$

Parameters are reported in Table 1 of the main paper.

Numerical Methods

The model was solved using the StarCCM+12.04. The two-dimensional cylindrical geometry was discretized using a directed mesh containing 95000 cells. A portion of the mesh is shown below: 


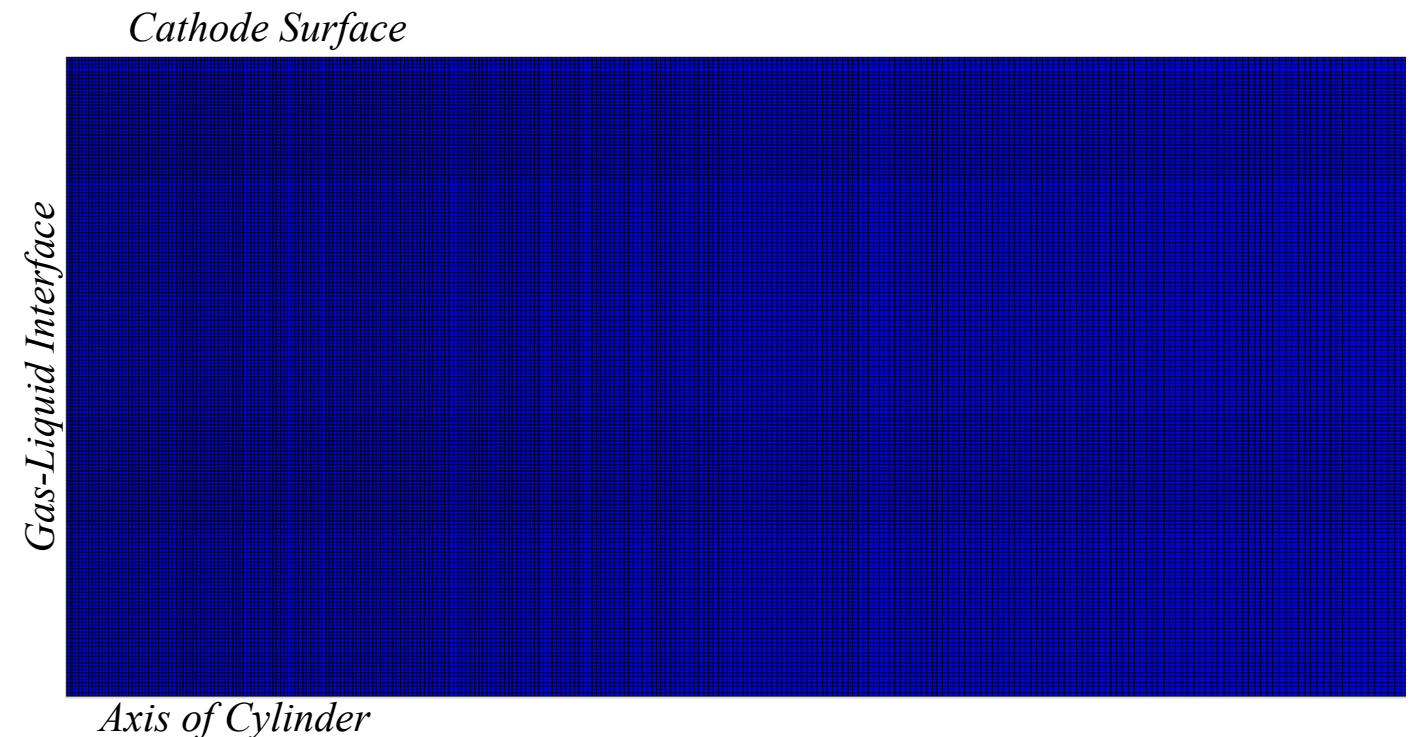

Figures S4: Mesh used for numerical solution of Multiphysics model. 


\section{Section S3: Resolved $\mathrm{CO}_{2}$ Pore Model}

A simpler, diffusion-reaction model, which described transport of $\mathrm{CO}_{2}$ molecules within a semi-infinite cylindrical pore, was also developed (see Figure S3). This model did not account for migration, as $\mathrm{CO}_{2}$ is a neutral molecule. It assumed that the $\mathrm{pH}$ is uniform within the catalyst pore, and ignored spatial variations in the overpotential on the catalyst surface. These simplifications allowed for non-dimensionalization and systematic analysis of the model. Under these conditions, the Nernst-Planck equation for $\mathrm{CO}_{2}$ reduces to:

$$
D \nabla^{2} c=k_{b} c
$$

where $c$ is the $\mathrm{CO}_{2}$ concentration, $D$ the $\mathrm{CO}_{2}$ diffusivity, and $k_{b}$ is a pseudofirst order rate constant related to the $\mathrm{OH}^{-}$concentration by:

$$
k_{b}=k_{2} c_{\mathrm{OH}^{-}} .
$$

On the catalyst surface, the Tafel condition reduces to a Robin condition with a first-order reaction,

$$
\left.J\right|_{\text {catalyst }}=\frac{\left.i\right|_{\text {catalyst }}}{n F}=-\left.D \frac{\partial c}{\partial x}\right|_{\text {catalyst }}=\left.k_{s} c\right|_{\text {catalyst }}
$$

Comparing Eq. (5) in the main paper and (3) leads to the following expression for the surface reaction rate constant:

$$
k_{s}=\frac{i_{0}^{\mathrm{COER}}}{n F c_{\mathrm{CO}_{2}}^{0}} \exp \left(-\frac{\alpha_{\mathrm{COER}} F}{R T} \eta\right) \text {. }
$$

As above, at the gas-liquid interface the $\mathrm{CO}_{2}$ concentration was specified:

$$
\left.c\right|_{\text {gas-liq. int. }}=c_{0} \text {. }
$$

This model was systematically compared with the more sophisticated multiphysics model over a range of pore radii, potentials and catalyst layer pHs (see section S7). The errors were small, especially in the bulk and

surface reaction controlled regime (average relative error: $1.6 \%$ ) in which the vast majority of practical systems operate. This is unsurprising, as spatial variations in $\mathrm{pH}$ and potential within a micron-scale catalyst layer are likely to be small. 


\section{Limiting Cases}

The pore models described above were compared with models for two limiting cases:

- TPR Control: When the surface reaction is fast and the pore radius is large, the $\mathrm{CO}_{2} \mathrm{R}$ reactions are concentrated in a narrow region on the catalyst adjacent to the TPBs.

- Uniform Radial Concentration: When the surface reaction is slow and the pore radius is small, the $\mathrm{CO}_{2}$ concentration within the pore is radially uniform.

Analytical expressions for the $\mathrm{CO}_{2}$ flux under these limiting conditions were derived, in order to aid the numerical development of operational phase diagrams describing transport processes at the pore scale.

\section{Idealized Model: TPR Control}

A simplified diffusion-reaction model of transport within the vicinity of a triple phase boundary was developed. The model was solved on a $2 \mathrm{D}$, semiinfinite square domain, approximating a 'corner' of a pore where the gas, liquid and solid phases meet at the TPB (see Figure 1c in the paper.) Similar to the resolved $\mathrm{CO}_{2}$ pore model described above, the model accounted for a first order reaction at the cathode surface and diffusion with pseudo-first order reaction within the bulk electrolyte:

$$
\begin{gathered}
D \nabla^{2} c=k_{b} c \\
\left.J\right|_{x=0}=-\left.D \frac{\partial c}{\partial x}\right|_{x=0}=\left.k_{s} c\right|_{x=0} \\
\left.c\right|_{y=0}=c_{0} .
\end{gathered}
$$

These PDEs were solved analytically via standard methods (see ESI, section S4), giving the following solution in dimensionless coordinates:

$$
\bar{c}(\bar{x}, \bar{y})=\int_{0}^{\infty}\left(1-e^{-\bar{x} \sqrt{\omega^{2}+1}}\left(\frac{L_{b} / L_{s}}{\sqrt{\omega^{2}+1}+L_{b} / L_{s}}\right)\right) \times \frac{2 \omega}{\pi\left(\omega^{2}+1\right)} \sin \omega \bar{y} \mathrm{~d} \omega
$$


The dimensionless variables and parameters are defined as follows:

$$
\begin{gathered}
\bar{c}=\frac{c}{c_{0}} ; \quad \bar{x}=\frac{x}{L_{b}} ; \quad \bar{y}=\frac{y}{L_{b}} \\
L_{b}=\sqrt{\frac{D}{k_{b}}} ; \quad L_{s}=\frac{D}{k_{s}} .
\end{gathered}
$$

$L_{b}$ and $L_{s}$ are characteristic length scales for diffusion with bulk reaction and diffusion with surface reaction, respectively.

The overall rate of reaction along the cathode surface may be calculated via the following integral:

$$
\dot{n}=\int_{0}^{\infty} k_{s} c\left(0, y ; L_{b} / L_{s}\right) \mathrm{d} y=c^{*} D\left(\frac{L_{b}}{L_{s}}\right) \int_{0}^{\infty} \bar{c}\left(0, \bar{y} ; L_{b} / L_{s}\right) \mathrm{d} \bar{y}
$$

Note that $\dot{n}$ has units mol m $\mathrm{m}^{-1} \mathrm{~s}^{-1}$, and represents the rate of $\mathrm{CO}_{2} \mathrm{R}$ per unit

length of TPB within the system. It may be related to the geometric partial current density for $\mathrm{CO}_{2} \mathrm{R}$ by:

$$
\frac{i}{n F}=\bar{L} \dot{n}
$$

where $\bar{L}$ is the length of TPBs per unit geometric area of the cathode.

By asymptotically approximating Eq. (12) near $L_{b} / L_{s}=0$ and $L_{b} / L_{s}=$ $\infty$, the following expressions may be derived for the $\mathrm{CO}_{2} \mathrm{R}$ partial current density in the limit of very slow and very fast surface reactions (see ESI, section S5):

$$
\frac{i}{n F c_{0} D \bar{L}}= \begin{cases}L_{b} / L_{s} & \text { when } L_{b} / L_{s} \ll 1 \\ 0.374+\frac{2}{\pi} \log L_{b} / L_{s} & \text { when } L_{b} / L_{s} \gg 1\end{cases}
$$

Finally, note that when $L_{b}=L_{s}$,

$$
i=0.63 n F c_{0} D \bar{L} \text {. }
$$

\section{Idealized Model: Uniform Radial Concentration}

When the surface reaction is slow and the pore radius is narrow, radial variations in concentration may be ignored. This limit is equivalent to the limit considered within the popular, homogenized GDE models of Weng et al. (Weng et al. 2018; Weng et al. 2019). In this limit, volume-averaging of 
the resolved $\mathrm{CO}_{2}$ model (Eq. (1) - (5) in section S3) eads to the following system of equations for the concentration of $\mathrm{CO}_{2}$ along the pore:

$$
\begin{gathered}
D \frac{\partial^{2} c}{\partial x^{2}}=\left(k_{b}+k_{s} a\right) c \\
\left.c\right|_{x=0}=c_{0}
\end{gathered}
$$

where $x$ is the distance along the pore, and $a$ is the specific surface area of the pore per unit volume. For a cylindrical pore,

$$
a=\frac{A}{V}=\frac{2 \pi R L}{\pi R^{2} L}=\frac{2}{R}
$$

where $R$ is the pore radius. Note that, for a liquid-filled, cylindrical pore,

$$
a=\bar{L} \text {. }
$$

Integration of these ODEs within a semi-infinite catalyst layer (assuming boundedness of $c$ as $x \rightarrow \infty$ ) gives the following concentration profile along the length of the pore:

$$
c=c_{0} \exp \left(-x\left(\frac{1}{L_{b}^{2}}+\frac{1}{{L_{s}^{\prime}}^{2}}\right)^{1 / 2}\right)
$$

where $L_{b}$ is as in Eq. (11) and $L_{s}^{\prime}$ is defined as

$$
L_{s}^{\prime}=\sqrt{\frac{D}{k_{s} a}} .
$$

In a system with narrow pores (so the concentration is radially uniform within each pore) $L_{s}^{\prime}$ represents the typical distance a $\mathrm{CO}_{2}$ molecule will diffuse before reacting on the catalyst surface. Note that Eq. (21) is similar to the definition of $L_{b}$ in Eq. (11), as, in the narrow-pore limit, catalyst reactions act as a homogenized $\mathrm{CO}_{2}$ sink term with rate constant $k_{s} a$.

The $\mathrm{CO}_{2} \mathrm{R}$ partial current density is given by:

$$
i_{1 D}=n F \int_{0}^{\infty} a k_{s} c(x) \mathrm{d} x
$$

Substituting in Eq. (20) gives:

$$
\frac{i_{1 D}}{n F a c_{0} k_{s}}=\left(\frac{1}{L_{b}^{2}}+\frac{1}{L_{s}^{\prime 2}}\right)^{-1 / 2}
$$


Note that $D$ refers to the diffusivity of $\mathrm{CO}_{2}$ in liquid water, in order that the volume-averaged 1D models may be directly compared with the resolved pore model. In an actual system, the finite porosity and tortuosity of the catalyst layer will reduce diffusive fluxes; these effects may be accounted for via a Bruggeman correction (Tjaden et al. 2016).

\section{Damköhler Numbers and Phase Diagram Development}

In the previous section, four characteristic lengthscales were introduced: $L_{b}, L_{s}, L_{s}^{\prime}$ and $1 / a$. The first three represent typical diffusion-reaction length scales under various limits, while the latter is proportional to the pore radius (Eq. (18)). These may be used to define Damköhler numbers for bulk and surface reaction:

$$
\mathrm{Da}_{\mathrm{b}} \equiv \frac{1 / a}{L_{b}}=\frac{1}{a} \sqrt{\frac{k_{b}}{D}} ; \quad \mathrm{Da}_{\mathrm{s}} \equiv \frac{1 / a}{L_{s}}=\frac{1}{a} \frac{k_{s}}{D} ;
$$

These Damköhler numbers determine the qualitative behaviour of transport processes within the catalyst pore, and the results of the models above may be formulated in terms of their relative magnitudes. In particular, the resolved $\mathrm{CO}_{2}$ pore model (Eq. (1) - (5) in section S3) may be non-dimensionalized, in order to express the $\mathrm{CO}_{2} \mathrm{R}$ partial current density as follows (see ESI, section S6):

$$
\frac{i_{\text {pore }}}{n F a c_{0} D}=f\left(\mathrm{Da}_{\mathrm{s}}, \mathrm{Da}_{\mathrm{b}}\right) \text {. }
$$

The narrow-pore limit, Eq. (23), in which transport is approximately one-dimensional, may be reformulated as:

$$
\frac{i_{1 \mathrm{D}}}{n F a c_{0} D}=h\left(\mathrm{Da}_{\mathrm{s}}, \mathrm{Da}_{\mathrm{b}}\right)=\left(\left(\frac{\mathrm{Da}_{\mathrm{b}}}{\mathrm{Da}_{\mathrm{s}}}\right)^{2}+\frac{1}{\mathrm{Da}_{\mathrm{s}}}\right)^{-1 / 2} .
$$

This narrow-pore limit may be further subdivided. When bulk reactions are much faster than surface reactions and control the depth of $\mathrm{CO}_{2}$ penetration into the pores, $L_{b} \ll L_{s}^{\prime}$, which implies $\left(\mathrm{Da}_{\mathrm{b}} / \mathrm{Da}_{\mathrm{s}}\right)^{2} \gg 1 / \mathrm{Da}_{\mathrm{s}}$, and Eq. (26) reduces to:

$$
\frac{i_{\mathrm{bulk}}}{n F a c_{0} D}=\left(\left(\frac{\mathrm{Da}_{\mathrm{b}}}{\mathrm{Da}_{\mathrm{s}}}\right)^{2}\right)^{-1 / 2}=\frac{\mathrm{Da}_{\mathrm{s}}}{\mathrm{Da}_{\mathrm{b}}}
$$


On the other hand, when surface reactions are fast enough to control the $\mathrm{CO}_{2}$ penetration depth (but not fast enough to radially deplete $\mathrm{CO}_{2}$ within the pores) $L_{s}^{\prime} \ll L_{b}$, and Eq. (26) reduces to:

$$
\frac{i_{\text {surf }}}{n F a c_{0} D}=\left(\frac{1}{\mathrm{Da}_{\mathrm{s}}}\right)^{-1 / 2}=\sqrt{\mathrm{Da}_{\mathrm{s}}}
$$

Similarly, the fast surface reaction limit of Eq. (14), in which transport within the TPR is rate controlling, is given by:

$$
\frac{i_{\mathrm{TPR}^{\prime}}}{n F a c_{0} D}=g\left(\mathrm{Da}_{\mathrm{s}}, \mathrm{Da}_{\mathrm{b}}\right)=0.374+\frac{2}{\pi} \log \frac{\mathrm{Da}_{\mathrm{s}}}{\mathrm{Da}_{\mathrm{b}}}
$$

The derivation of this expression involved modelling the TPR on a semiinfinite quarter-plane, so this approximation is only reasonable when the pore radius is large, and in particular when $1 / a \gg L_{b}$. When $1 / a \ll L_{b}$, bulk reactions will not influence transport near the TPB, and we would expect the current density across the TPB to scale with $(1 / a) / L_{s}$ rather than $L_{b} / L_{s}$, in which case Eq. (14) becomes:

$$
\frac{i_{\mathrm{TPR}^{\prime \prime}}}{n F a c_{0} D}=0.374+\frac{2}{\pi} \log \mathrm{Da}_{\mathrm{s}}
$$

Both expression are captured by the more general limiting expression:

$$
\frac{i_{\mathrm{TPR}}}{n F a c_{0} D}=0.374+\frac{2}{\pi} \log \left(\min \left(\frac{\mathrm{Da}_{\mathrm{s}}}{\mathrm{Da}_{\mathrm{b}}}, \mathrm{Da}_{\mathrm{s}}\right)\right)
$$

An operational phase diagram was numerically created by systematically varying $\mathrm{Da}_{\mathrm{s}}$ and $\mathrm{Da}_{\mathrm{b}}$ and identifying locations within the parameter space for which:

$$
\left|\frac{i_{\text {pore }}-i_{\text {lim }}}{i_{\text {pore }}}\right|<\varepsilon=0.1
$$

where $\varepsilon$ is a tolerance, and $i_{\text {lim }}$ refers to the various limiting cases outlined above. This procedure allowed the automatic mapping of regions of phase space in which catalyst performance was controlled by transport across local TPBs, and in which transport was approximately one-dimensional within the pores. These numerical results were compared with theoretical predictions 
based on the relative magnitudes of $L_{s}, L_{b}, L_{s}^{\prime}$ and $1 / a$. These calculations also facilitated the evaluation of an effectiveness factor, (Bird et al. 2006)

$$
\xi \equiv \frac{i_{\text {pore }}}{i_{1 \mathrm{D}}}
$$

which allows radial concentration variations to be accounted for in a volumeaveraged model.

\section{Section S4: Analytical Solution}

In what follows, we solve a steady state diffusion-reaction equation on the quarter plane, with an inhomogeneous Dirichlet condition on one boundary, and a homogeneous Robin boundary condition on the other. Note that in this section the $x$ and $y$ axes are inverted relative to the images presented in the paper.

$$
\begin{gathered}
\nabla^{2} u=\xi u ; \quad x>0, y>0 \\
\frac{\partial u}{\partial y}(x, 0)=\kappa u(x, 0) ; \quad x>0 \\
u(0, y)=g(y) ; \quad y>0
\end{gathered}
$$

We now solve this problem using a sine-transform in $x$ (see Haberman (2003)). Multiply the equation by $\sin \omega x$ and integrate from $x=0$ to $\infty$ :

$$
\begin{gathered}
\int_{0}^{\infty} \frac{\partial^{2} u}{\partial x^{2}} \sin \omega x \mathrm{~d} x+\int_{0}^{\infty} \frac{\partial^{2} u}{\partial y^{2}} \sin \omega x \mathrm{~d} x=\xi \int_{0}^{\infty} u \sin \omega x \mathrm{~d} x \\
\left.\frac{\partial u}{\partial x} \sin \omega x\right|_{x=0} ^{\infty}-\omega \int_{0}^{\infty} \frac{\partial u}{\partial x} \cos \omega x \mathrm{~d} x+\frac{\partial^{2}}{\partial y^{2}}\left[\int_{0}^{\infty} u \sin \omega x \mathrm{~d} x\right]=\xi \int_{0}^{\infty} u \sin \omega x \mathrm{~d} x
\end{gathered}
$$

As $\sin 0=0$ and $\partial u / \partial x \rightarrow 0$ as $x \rightarrow \infty$, we can drop the first term:

$$
-\omega \int_{0}^{\infty} \frac{\partial u}{\partial x} \cos \omega x \mathrm{~d} x+\frac{\partial^{2}}{\partial y^{2}}\left[\int_{0}^{\infty} u \sin \omega x \mathrm{~d} x\right]=\xi \int_{0}^{\infty} u \sin \omega x \mathrm{~d} x
$$

Applying integration by parts again,

$-\left.u \omega \cos \omega x\right|_{x=0} ^{\infty}-\omega^{2} \int_{0}^{\infty} u \sin \omega x \mathrm{~d} x+\frac{\partial^{2}}{\partial y^{2}}\left[\int_{0}^{\infty} u \sin \omega x \mathrm{~d} x\right]=\xi \int_{0}^{\infty} u \sin \omega x \mathrm{~d} x$ 
The first term does not cancel, as $u(0, y) \omega \cos (0)=\omega g(y)$. The term at $\infty$ will equal zero provided that $g(y) \rightarrow 0$ as $y \rightarrow \infty$. We then have

$$
\omega g(y)-\omega^{2} \int_{0}^{\infty} u \sin \omega x \mathrm{~d} x+\frac{\partial^{2}}{\partial y^{2}}\left[\int_{0}^{\infty} u \sin \omega x \mathrm{~d} x\right]=\xi \int_{0}^{\infty} u \sin \omega x \mathrm{~d} x
$$

Let $U$ be the sine Fourier transform of $u$ in the variable $x$ :

$$
U(\omega, y) \equiv \frac{2}{\pi} \int_{0}^{\infty} u \sin \omega x \mathrm{~d} x
$$

Then we have:

$$
\begin{gathered}
\frac{\partial^{2}}{\partial y^{2}}\left[\frac{2}{\pi} \int_{0}^{\infty} u \sin \omega x \mathrm{~d} x\right]-\left(\omega^{2}+\xi\right) \frac{2}{\pi} \int_{0}^{\infty} u \sin \omega x \mathrm{~d} x=-\frac{2}{\pi} \omega g(y) . \\
\frac{\partial^{2}}{\partial y^{2}} U(\omega, y)-\left(\omega^{2}+\xi\right) U(\omega, y)=-\frac{2}{\pi} \omega g(y) .
\end{gathered}
$$

We now need to solve this ODE in $y$ subject to the boundary conditions at $y=0$ and $y \rightarrow \infty$. If $g(y) \rightarrow 0$ as $y \rightarrow \infty$, then we also expect $u(y) \rightarrow 0$ and

$$
\lim _{y \rightarrow \infty} U(\omega, y)=0
$$

On the other hand, if $\partial u / \partial y=\kappa u$ at $y=0$, we expect,

$$
\left.\frac{\partial U}{\partial y}\right|_{y=0}-\left.\kappa U\right|_{y=0}=\frac{2}{\pi} \int_{0}^{\infty}\left[\left.\frac{\partial u}{\partial y}\right|_{y=0}-\left.\kappa u\right|_{y=0}\right] \sin \omega x \mathrm{~d} x=0
$$

Hence, our boundary conditions are

$$
U(\omega, \infty)=0 ; \quad \frac{\partial U}{\partial y}(\omega, 0)=\kappa U(\omega, 0) .
$$

We begin by solving the homogeneous problem,

$$
\frac{\partial^{2}}{\partial y^{2}} U(\omega, y)-\left(\omega^{2}+\xi\right) U(\omega, y)=0 .
$$

This has the general solution:

$$
U_{0}=A(\omega) e^{\sqrt{\omega^{2}+\xi} y}+B(\omega) e^{-\sqrt{\omega^{2}+\xi} y} .
$$


We also need a particular solution to the non-homogeneous equation. It is possible to show by the method of variation of parameters that the following is a particular solution:

$$
U_{p}=-U_{1} \int \frac{U_{2} F(y)}{W} d y+U_{2} \int \frac{U_{1} F(y)}{W} d y
$$

where $U_{1}$ and $U_{2}$ are two linearly independent solutions of the homogeneous problem, and $F(y)$ is the right hand side of the ODE; in this case

$$
F(y)=-\frac{2}{\pi} \omega g(y) ; \quad U_{1}=e^{\sqrt{\omega^{2}+\xi} y} ; \quad U_{2}=e^{-\sqrt{\omega^{2}+\xi} y}
$$

$W$ is the Wronskian,

$$
\begin{aligned}
W & =U_{1} U_{2}^{\prime}-U_{2} U_{1}^{\prime} \\
& =e^{\sqrt{\omega^{2}+\xi} y}\left(-\sqrt{\omega^{2}+\xi} e^{-\sqrt{\omega^{2}+\xi} y}\right)-e^{-\sqrt{\omega^{2}+\xi} y}\left(\sqrt{\omega^{2}+\xi} e^{\sqrt{\omega^{2}+\xi} y}\right) \\
& =-2 \sqrt{\omega^{2}+\xi}
\end{aligned}
$$

We therefore have

$$
\begin{gathered}
U_{p}=\frac{-e^{\sqrt{\omega^{2}+\xi} y}}{-2 \sqrt{\omega^{2}+\xi}} \int_{0}^{y} e^{-\sqrt{\omega^{2}+\xi} y}\left(-\frac{2 \omega g(y)}{\pi}\right) \mathrm{d} y \\
+\frac{e^{-\sqrt{\omega^{2}+\xi} y}}{-2 \sqrt{\omega^{2}+\xi}} \int_{0}^{y} e^{\sqrt{\omega^{2}+\xi} y}\left(-\frac{2 \omega g(y)}{\pi}\right) \mathrm{d} y \\
U_{p}=-\frac{\omega e^{\sqrt{\omega^{2}+\xi} y}}{\pi \sqrt{\omega^{2}+\xi}} \int_{0}^{y} e^{-\sqrt{\omega^{2}+\xi} y} g(y) \mathrm{d} y+\frac{\omega e^{-\sqrt{\omega^{2}+\xi} y}}{\pi \sqrt{\omega^{2}+\xi}} \int_{0}^{y} e^{\sqrt{\omega^{2}+\xi} y} g(y) \mathrm{d} y
\end{gathered}
$$

We now double check that this particular solution is indeed a solution to the pde's

$$
\begin{gathered}
U_{p}=-\frac{\omega e^{\sqrt{\omega^{2}+\xi} y}}{\pi \sqrt{\omega^{2}+\xi}} \int_{0}^{y} e^{-\sqrt{\omega^{2}+\xi} y} g(y) \mathrm{d} y+\frac{\omega e^{-\sqrt{\omega^{2}+\xi} y}}{\pi \sqrt{\omega^{2}+\xi}} \int_{0}^{y} e^{\sqrt{\omega^{2}+\xi} y} g(y) \mathrm{d} y \\
\frac{\partial U_{p}}{\partial y}=-\frac{\omega e^{\sqrt{\omega^{2}+\xi} y}}{\pi} \int_{0}^{y} e^{-\sqrt{\omega^{2}+\xi} y} g(y) \mathrm{d} y-\frac{\omega e^{-\sqrt{\omega^{2}+\xi} y}}{\pi} \int_{0}^{y} e^{\sqrt{\omega^{2}+\xi} y} g(y) \mathrm{d} y \\
-\frac{\omega e^{\sqrt{\omega^{2}+\xi} y}}{\pi \sqrt{\omega^{2}+\xi}} e^{-\sqrt{\omega^{2}+\xi} y} g(y)+\frac{\omega e^{-\sqrt{\omega^{2}+\xi} y}}{\pi \sqrt{\omega^{2}+\xi}} e^{\sqrt{\omega^{2}+\xi} y} g(y)
\end{gathered}
$$




$$
\frac{\partial U_{p}}{\partial y}=-\frac{\omega e^{-\sqrt{\omega^{2}+\xi} y}}{\pi} \int_{0}^{y} e^{\sqrt{\omega^{2}+\xi} y} g(y) \mathrm{d} y-\frac{\omega e^{\sqrt{\omega^{2}+\xi} y}}{\pi} \int_{0}^{y} e^{-\sqrt{\omega^{2}+\xi} y} g(y) \mathrm{d} y
$$

And then

$$
\begin{aligned}
\frac{\partial^{2} U_{p}}{\partial y^{2}}= & \frac{\omega \sqrt{\omega^{2}+\xi} e^{-\sqrt{\omega^{2}+\xi} y}}{\pi} \int_{0}^{y} e^{\sqrt{\omega^{2}+\xi} y} g(y) \mathrm{d} y \\
& -\frac{\omega \sqrt{\omega^{2}+\xi} e^{\sqrt{\omega^{2}+\xi} y}}{\pi} \int_{0}^{y} e^{-\sqrt{\omega^{2}+\xi} y} g(y) \mathrm{d} y \\
& -\frac{\omega e^{-\sqrt{\omega^{2}+\xi} y}}{\pi} e^{\sqrt{\omega^{2}+\xi} y} g(y)-\frac{\omega e^{\sqrt{\omega^{2}+\xi} y}}{\pi} e^{-\sqrt{\omega^{2}+\xi} y} g(y) \\
\frac{\partial^{2} U_{p}}{\partial y^{2}}= & \frac{\omega \sqrt{\omega^{2}+\xi} e^{-\sqrt{\omega^{2}+\xi} y}}{\pi} \int_{0}^{y} e^{\sqrt{\omega^{2}+\xi} y} g(y) \mathrm{d} y \\
& -\frac{\omega \sqrt{\omega^{2}+\xi} e^{\sqrt{\omega^{2}+\xi} y}}{\pi} \int_{0}^{y} e^{-\sqrt{\omega^{2}+\xi} y} g(y) \mathrm{d} y-\frac{2 \omega g(y)}{\pi}
\end{aligned}
$$

and clearly we have:

$$
\frac{\partial^{2} U_{p}}{\partial y^{2}}=\left(\omega^{2}+\xi\right) U_{p}(\omega, y)-\frac{2 \omega g(y)}{\pi}
$$

as required. The general solution is therefore:

$$
\begin{gathered}
U=U_{p}+U_{0} \\
U=\frac{\omega e^{-\sqrt{\omega^{2}+\xi} y}}{\pi \sqrt{\omega^{2}+\xi}} \int_{0}^{y} e^{\sqrt{\omega^{2}+\xi} y} g(y) \mathrm{d} y-\frac{\omega e^{\sqrt{\omega^{2}+\xi} y}}{\pi \sqrt{\omega^{2}+\xi}} \int_{0}^{y} e^{-\sqrt{\omega^{2}+\xi} y} g(y) \mathrm{d} y \\
+A(\omega) e^{\sqrt{\omega^{2}+\xi} y}+B(\omega) e^{-\sqrt{\omega^{2}+\xi} y} .
\end{gathered}
$$

The first boundary condition implies:

$$
\left.\frac{\partial U}{\partial y}\right|_{y=0}=\left.\kappa U\right|_{y=0}
$$


It is clear from above that $U_{p}(\omega, 0)=\partial U_{p} / \partial y(\omega, 0)=0$, and so this condition reduces to:

$$
\left.\frac{\partial U_{0}}{\partial y}\right|_{y=0}=\left.\kappa U_{0}\right|_{y=0}
$$

As

$$
U_{0}=A(\omega) e^{\sqrt{\omega^{2}+\xi} y}+B(\omega) e^{-\sqrt{\omega^{2}+\xi} y}
$$

this reduces to:

$$
\begin{gathered}
\sqrt{\omega^{2}+\xi} A(\omega)-\sqrt{\omega^{2}+\xi} B(\omega)=\kappa(A(\omega)+B(\omega)) \\
B(\omega)=A(\omega)\left(\frac{\sqrt{\omega^{2}+\xi}-\kappa}{\sqrt{\omega^{2}+\xi}+\kappa}\right)
\end{gathered}
$$

The above may then be written:

$$
\begin{aligned}
U= & \frac{\omega e^{-\sqrt{\omega^{2}+\xi} y}}{\pi \sqrt{\omega^{2}+\xi}} \int_{0}^{y} e^{\sqrt{\omega^{2}+\xi} y} g(y) \mathrm{d} y-\frac{\omega e^{\sqrt{\omega^{2}+\xi} y}}{\pi \sqrt{\omega^{2}+\xi}} \int_{0}^{y} e^{-\sqrt{\omega^{2}+\xi} y} g(y) \mathrm{d} y \\
& +A(\omega) e^{\sqrt{\omega^{2}+\xi} y}+B(\omega) e^{-\sqrt{\omega^{2}+\xi} y} . \\
U= & e^{\sqrt{\omega^{2}+\xi} y}\left(A(\omega)-\frac{\omega}{\pi \sqrt{\omega^{2}+\xi}} \int_{0}^{y} e^{-\sqrt{\omega^{2}+\xi} y^{\prime}} g\left(y^{\prime}\right) \mathrm{d} y^{\prime}\right) \\
& +e^{-\sqrt{\omega^{2}+\xi} y}\left(B(\omega)+\frac{\omega}{\pi \sqrt{\omega^{2}+\xi}} \int_{0}^{y} e^{\sqrt{\omega^{2}+\xi} y^{\prime}} g\left(y^{\prime}\right) \mathrm{d} y^{\prime}\right) \\
U= & e^{\sqrt{\omega^{2}+\xi} y}\left(A(\omega)-\frac{\omega}{\pi \sqrt{\omega^{2}+\xi}} \int_{0}^{y} e^{-\sqrt{\omega^{2}+\xi} y^{\prime}} g\left(y^{\prime}\right) \mathrm{d} y^{\prime}\right. \\
+ & e^{-\sqrt{\omega^{2}+\xi} y}\left(A(\omega)\left(\frac{\sqrt{\omega^{2}+\xi}-\kappa}{\sqrt{\omega^{2}+\xi}+\kappa}\right)+\frac{\omega}{\pi \sqrt{\omega^{2}+\xi}} \int_{0}^{y} e^{\sqrt{\omega^{2}+\xi} y^{\prime}} g\left(y^{\prime}\right) \mathrm{d} y^{\prime}\right)
\end{aligned}
$$

The second boundary condition is that

$$
U(\omega, \infty)=0
$$


The second term will go to zero as $y \rightarrow \infty$, provided that $g(y) \rightarrow 0$ relatively quickly. On the other hand, the first term will only go to zero if

$$
A(\omega)=\frac{\omega}{\pi \sqrt{\omega^{2}+\xi}} \int_{0}^{\infty} e^{-\sqrt{\omega^{2}+\xi} y^{\prime}} g\left(y^{\prime}\right) \mathrm{d} y^{\prime}
$$

Substituting this in gives the following solution:

$$
\begin{aligned}
U= & e^{\sqrt{\omega^{2}+\xi} y}\left(\frac{\omega}{\pi \sqrt{\omega^{2}+\xi}} \int_{0}^{\infty} e^{-\sqrt{\omega^{2}+\xi} y^{\prime}} g\left(y^{\prime}\right) \mathrm{d} y^{\prime}\right. \\
& \left.-\frac{\omega}{\pi \sqrt{\omega^{2}+\xi}} \int_{0}^{y} e^{-\sqrt{\omega^{2}+\xi} y^{\prime}} g\left(y^{\prime}\right) \mathrm{d} y^{\prime}\right) \\
+ & e^{-\sqrt{\omega^{2}+\xi} y}\left(\left(\frac{\sqrt{\omega^{2}+\xi}-\kappa}{\sqrt{\omega^{2}+\xi}+\kappa}\right) \frac{\omega}{\pi \sqrt{\omega^{2}+\xi}} \int_{0}^{\infty} e^{-\sqrt{\omega^{2}+\xi} y^{\prime}} g\left(y^{\prime}\right) \mathrm{d} y^{\prime}\right. \\
& \left.+\frac{\omega}{\pi \sqrt{\omega^{2}+\xi}} \int_{0}^{y} e^{\sqrt{\omega^{2}+\xi} y^{\prime}} g\left(y^{\prime}\right) \mathrm{d} y^{\prime}\right)
\end{aligned}
$$

The next step is to invert the Fourier Transform, in order to calculate $u(x, y)$ :

$$
u(x, y)=\int_{0}^{\infty} U(\omega, y) \sin \omega x \mathrm{~d} \omega
$$

Let's substitute our expression for $U$ into this:

$$
\begin{array}{r}
u(x, y)=\int_{0}^{\infty}\left[e ^ { \sqrt { \omega ^ { 2 } + \xi } y } \left(\frac{\omega}{\pi \sqrt{\omega^{2}+\xi}} \int_{0}^{\infty} e^{-\sqrt{\omega^{2}+\xi} y^{\prime}} g\left(y^{\prime}\right) \mathrm{d} y^{\prime}\right.\right. \\
\left.-\frac{\omega}{\pi \sqrt{\omega^{2}+\xi}} \int_{0}^{y} e^{-\sqrt{\omega^{2}+\xi} y^{\prime}} g\left(y^{\prime}\right) \mathrm{d} y^{\prime}\right) \\
+e^{-\sqrt{\omega^{2}+\xi} y}\left(\left(\frac{\sqrt{\omega^{2}+\xi}-\kappa}{\sqrt{\omega^{2}+\xi}+\kappa}\right) \frac{\omega}{\pi \sqrt{\omega^{2}+\xi}} \int_{0}^{\infty} e^{-\sqrt{\omega^{2}+\xi} y^{\prime}} g\left(y^{\prime}\right) \mathrm{d} y^{\prime}\right. \\
\left.\left.+\frac{\omega}{\pi \sqrt{\omega^{2}+\xi}} \int_{0}^{y} e^{\sqrt{\omega^{2}+\xi} y^{\prime}} g\left(y^{\prime}\right) \mathrm{d} y^{\prime}\right)\right] \sin \omega x \mathrm{~d} \omega
\end{array}
$$




$$
\begin{array}{r}
u(x, y)=\int_{0}^{\infty}\left[e^{\sqrt{\omega^{2}+\xi} y}\left(\frac{\omega}{\pi \sqrt{\omega^{2}+\xi}} \int_{y}^{\infty} e^{-\sqrt{\omega^{2}+\xi} y^{\prime}} g\left(y^{\prime}\right) \mathrm{d} y^{\prime}\right)\right. \\
+e^{-\sqrt{\omega^{2}+\xi} y}\left(\left(\frac{\sqrt{\omega^{2}+\xi}-\kappa}{\sqrt{\omega^{2}+\xi}+\kappa}\right) \frac{\omega}{\pi \sqrt{\omega^{2}+\xi}} \int_{0}^{\infty} e^{-\sqrt{\omega^{2}+\xi} y^{\prime}} g\left(y^{\prime}\right) \mathrm{d} y^{\prime}\right. \\
\left.\left.\quad+\frac{\omega}{\pi \sqrt{\omega^{2}+\xi}} \int_{0}^{y} e^{\sqrt{\omega^{2}+\xi} y^{\prime}} g\left(y^{\prime}\right) \mathrm{d} y^{\prime}\right)\right] \sin \omega x \mathrm{~d} \omega
\end{array}
$$

Breaking this into three integrals, and swapping the order of integration,

$$
\begin{aligned}
& u(x, y)=\int_{y}^{\infty} g\left(y^{\prime}\right) \int_{0}^{\infty} \frac{\omega}{\pi \sqrt{\omega^{2}+\xi}} e^{-\sqrt{\omega^{2}+\xi}\left(y^{\prime}-y\right)} \sin \omega x \mathrm{~d} \omega \mathrm{d} y^{\prime} \\
& +\int_{0}^{y} g\left(y^{\prime}\right) \int_{0}^{\infty} \frac{\omega}{\pi \sqrt{\omega^{2}+\xi}} e^{-\sqrt{\omega^{2}+\xi}\left(y-y^{\prime}\right)} \sin \omega x \mathrm{~d} \omega \mathrm{d} y^{\prime} \\
& \quad+\int_{0}^{\infty} g\left(y^{\prime}\right)\left[\int_{0}^{\infty}\left(\frac{\sqrt{\omega^{2}+\xi}-\kappa}{\sqrt{\omega^{2}+\xi}+\kappa}\right) \frac{\omega}{\pi \sqrt{\omega^{2}+\xi}} e^{-\sqrt{\omega^{2}+\xi}\left(y+y^{\prime}\right)} \sin \omega x \mathrm{~d} \omega\right] \mathrm{d} y^{\prime}
\end{aligned}
$$

$$
\begin{aligned}
& u(x, y)=\int_{0}^{\infty} g\left(y^{\prime}\right) \int_{0}^{\infty} \frac{\omega}{\pi \sqrt{\omega^{2}+\xi}} e^{-\sqrt{\omega^{2}+\xi}\left|y^{\prime}-y\right|} \sin \omega x \mathrm{~d} \omega \mathrm{d} y^{\prime} \\
& \quad+\int_{0}^{\infty} g\left(y^{\prime}\right)\left[\int_{0}^{\infty}\left(\frac{\sqrt{\omega^{2}+\xi}-\kappa}{\sqrt{\omega^{2}+\xi}+\kappa}\right) \frac{\omega}{\pi \sqrt{\omega^{2}+\xi}} e^{-\sqrt{\omega^{2}+\xi}\left(y+y^{\prime}\right)} \sin \omega x \mathrm{~d} \omega\right] \mathrm{d} y^{\prime}
\end{aligned}
$$

$$
\begin{aligned}
u(x, y)=\int_{0}^{\infty} & g\left(y^{\prime}\right)\left[\int _ { 0 } ^ { \infty } \left(e^{-\sqrt{\omega^{2}+\xi}\left|y^{\prime}-y\right|}\right.\right. \\
& \left.\left.+\left(\frac{\sqrt{\omega^{2}+\xi}-\kappa}{\sqrt{\omega^{2}+\xi}+\kappa}\right) e^{-\sqrt{\omega^{2}+\xi}\left(y+y^{\prime}\right)}\right) \frac{\omega}{\pi \sqrt{\omega^{2}+\xi}} \sin \omega x \mathrm{~d} \omega\right] \mathrm{d} y^{\prime}
\end{aligned}
$$

This is the general solution to the problem on the quarter plane. We are interested in the solution along the $x$-axis $(y=0)$ when $g(y)=1$. This is: 


$$
\begin{gathered}
u(x, 0)=\frac{1}{\pi} \int_{0}^{\infty}\left[\int_{0}^{\infty}\left(e^{-\sqrt{\omega^{2}+\xi} y^{\prime}}+\left(\frac{\sqrt{\omega^{2}+\xi}-\kappa}{\sqrt{\omega^{2}+\xi}+\kappa}\right) e^{-\sqrt{\omega^{2}+\xi} y^{\prime}}\right) \frac{\omega}{\sqrt{\omega^{2}+\xi}} \sin \omega x \mathrm{~d} \omega\right] \mathrm{d} y^{\prime} \\
u(x, 0)=\frac{1}{\pi} \int_{0}^{\infty}\left[\int_{0}^{\infty}\left(\left(\frac{2 \sqrt{\omega^{2}+\xi}}{\sqrt{\omega^{2}+\xi}+\kappa}\right) e^{-\sqrt{\omega^{2}+\xi} y^{\prime}}\right) \frac{\omega}{\sqrt{\omega^{2}+\xi}} \sin \omega x \mathrm{~d} \omega\right] \mathrm{d} y^{\prime} \\
u(x, 0)=\frac{1}{\pi} \int_{0}^{\infty}\left[\int_{0}^{\infty} \frac{2 \omega}{\sqrt{\omega^{2}+\xi}+\kappa} e^{-\sqrt{\omega^{2}+\xi} y^{\prime}} \sin \omega x \mathrm{~d} \omega\right] \mathrm{d} y^{\prime}
\end{gathered}
$$

In the physical problem, $\kappa=k_{s} / \mathcal{D}$ and $\xi=k_{b} / \mathcal{D}$, while $c(x=0)=c^{*}$. Substituting these in gives,

$$
c(x, 0)=\frac{c^{*}}{\pi} \int_{0}^{\infty}\left[\int_{0}^{\infty} \frac{2 \omega}{\sqrt{\omega^{2}+k_{b} / \mathcal{D}}+k_{s} / \mathcal{D}} e^{-\sqrt{\omega^{2}+k_{b} / \mathcal{D}} y^{\prime}} \sin \omega x \mathrm{~d} \omega\right] \mathrm{d} y^{\prime}
$$

In dimensionless form, Eq. (12) - (14) in the main paper reduce to

$$
\begin{gathered}
\nabla^{2} \bar{c}=\bar{c} \\
\left.\frac{\partial \bar{c}}{\partial x}\right|_{\bar{x}=0}=\left.\left(L_{b} / L_{s}\right) \bar{c}\right|_{\bar{x}=0} \\
\left.\bar{c}\right|_{\bar{y}=0}=1
\end{gathered}
$$

where

$$
\begin{gathered}
\bar{c}=\frac{c}{c^{*}} ; \quad \bar{x}=\frac{x}{L_{b}} ; \quad \bar{y}=\frac{y}{L_{b}} \\
L_{b}=\sqrt{\frac{D}{k_{b}}} ; \quad L_{s}=\frac{D}{k_{s}}
\end{gathered}
$$

The analytical solution above then reduces to:

$$
\begin{aligned}
& \bar{c}(\bar{x}, \bar{y})=\frac{1}{\pi} \int_{0}^{\infty}\left[\int _ { 0 } ^ { \infty } \left(e^{-\sqrt{\omega^{2}+1}\left|\bar{x}^{\prime}-\bar{x}\right|}\right.\right. \\
&\left.+\left(\frac{\sqrt{\omega^{2}+1}-L_{b} / L_{s}}{\sqrt{\omega^{2}+1}+L_{b} / L_{s}}\right) e^{-\sqrt{\omega^{2}+1}\left(\bar{x}^{\prime}+\bar{x}\right)}\right) \times \\
&\left.\frac{\omega}{\sqrt{\omega^{2}+1}} \sin \omega \bar{y} \mathrm{~d} \omega\right] \mathrm{d} \bar{x}^{\prime}
\end{aligned}
$$


By swapping the order of integration, this may be reduced to a single integral expression, given in Eq. (15) of the main text:

$$
\bar{c}(\bar{x}, \bar{y})=\int_{0}^{\infty}\left(1-e^{-\bar{x} \sqrt{\omega^{2}+1}}\left(\frac{L_{b} / L_{s}}{\sqrt{\omega^{2}+1}+L_{b} / L_{s}}\right)\right) \times \frac{2 \omega}{\pi\left(\omega^{2}+1\right)} \sin \omega \bar{y} \mathrm{~d} \omega
$$

\section{Section S5: Scaling at Large $\lambda$}

Numerical evaluation of the analytical solution suggests that $\dot{n}$ becomes a linear function of $\log k_{s}$ when $k_{s} / \sqrt{\mathcal{D} k_{b}} \gg 1$ (c.f. Figure 2c of the main paper.) In this section, we will show that the concentration profiles on the cathode surface (c.f. Figure $2 \mathrm{~b}$ of the main paper) asymptotically scale as $\bar{c} \propto$ $1 / \bar{x}$ for large values of $\lambda$. The integration of these profiles in the calculation of $\dot{n}$ then leads to the logarithmic scaling law.

For large values of $\lambda$, when the surface reaction is very fast, it is simplest to analyze the problem via the alternative set of dimensionless variables, in which $x$ and $y$ are scaled via $L_{s}$ instead of $L_{b}$ :

$$
\bar{c}=\frac{c}{c^{*}} ; \quad \bar{x}=\frac{x}{L_{s}} ; \quad \bar{y}=\frac{y}{L_{s}} \quad \lambda=\frac{k_{s}}{\sqrt{k_{b} \mathcal{D}}}=\frac{L_{b}}{L_{s}}
$$

With these dimensionless variables, Eq. (12) - (14) in the main paper reduce to

$$
\begin{gathered}
\nabla^{2} \bar{c}=\lambda^{-2} \bar{c} \\
\left.\frac{\partial \bar{c}}{\partial x}\right|_{\bar{x}=0}=\left.\bar{c}\right|_{\bar{x}=0} \\
\left.\bar{c}\right|_{\bar{y}=0}=1
\end{gathered}
$$

and the analytical solution along the cathode reduces to

$$
\bar{c}(0, \bar{y})=\frac{1}{\pi} \int_{0}^{\infty}\left[\int_{0}^{\infty} \frac{2 \omega}{\sqrt{\omega^{2}+\lambda^{-2}}+1} e^{-\sqrt{\omega^{2}+\lambda^{-2}} x^{\prime}} \sin \omega \bar{y} \mathrm{~d} \omega\right] \mathrm{d} x^{\prime}
$$

In the limit in which $\lambda \rightarrow \infty$, this reduces to

$$
\bar{c}(0, \bar{y})=\frac{1}{\pi} \int_{0}^{\infty}\left[\int_{0}^{\infty} \frac{2 \omega}{\omega+1} e^{-\omega x^{\prime}} \sin \omega \bar{y} \mathrm{~d} \omega\right] \mathrm{d} x^{\prime}
$$


This simpler expression may be integrated analytically, giving the following solution in the limit of very fast surface reaction $(\lambda \rightarrow \infty)$,

$$
\bar{c}(0, \bar{y})=\frac{2}{\pi} \operatorname{Ci}(\bar{y}) \sin (\bar{y})-\frac{2}{\pi} \operatorname{Si}(\bar{y}) \cos (\bar{y})+\cos (\bar{y})
$$

where $\mathrm{Ci}$ and $\mathrm{Si}$ are the cosine and sine integral functions, respectively. This function goes to 0 as $\bar{y} \rightarrow \infty$, and taking the Taylor series at $\bar{y}=\infty$ reveals a $1 / \bar{y}$ decay of the form:

$$
\bar{c}(\bar{y})=\frac{2}{\pi} \frac{1}{\bar{y}}+\mathcal{O}\left(\frac{1}{\bar{y}^{3}}\right) \quad \text { as } \bar{y} \rightarrow \infty
$$

The asymptotic expression, Eq. (92) and the analytical expression, Eq. (91), are plotted in the figure below.

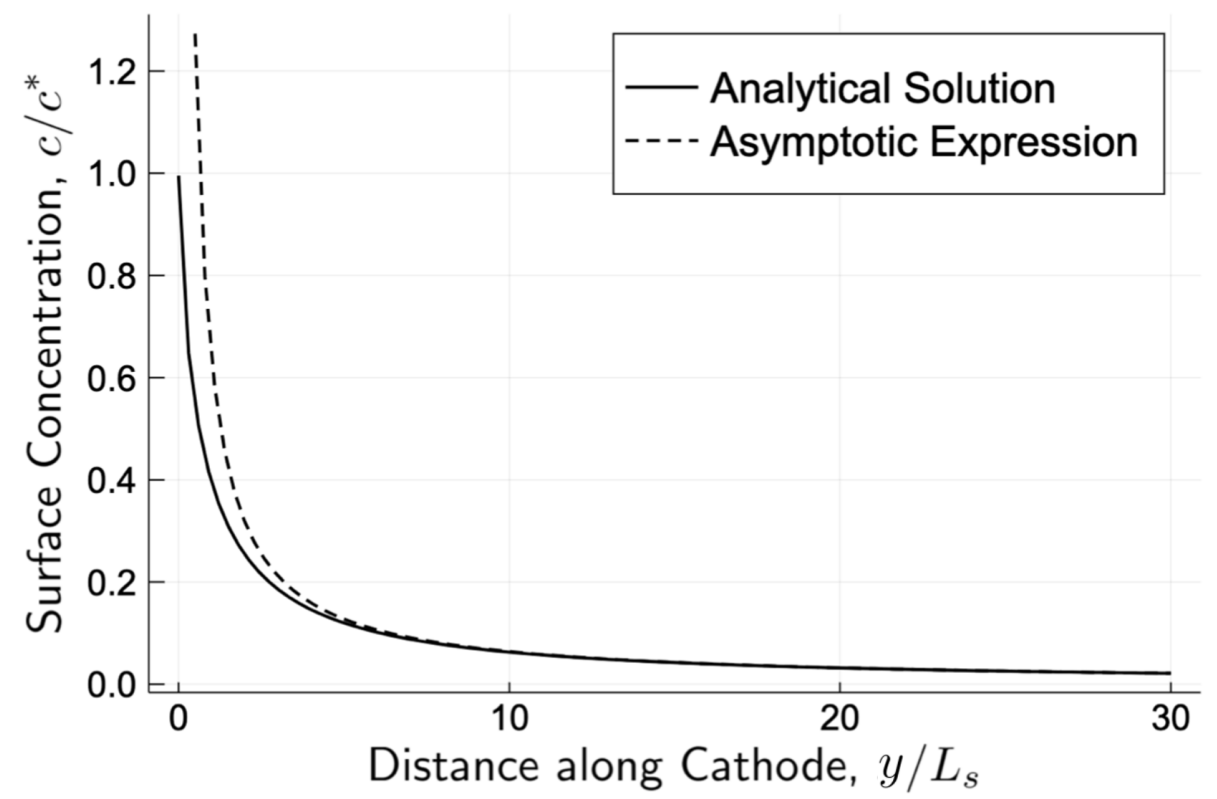

Figures S5: Surface concentration along cathode.

The rate of mass transfer within the TPR is given by:

$$
\dot{n}=\int_{0}^{\infty} k_{s} c(y) \mathrm{d} y=c^{*} \mathcal{D} \int_{0}^{\infty} \bar{c}(\bar{y}) \mathrm{d} \bar{y}
$$


Assuming that the asymptotic approximation is reasonable above some large value $\bar{y}=\bar{y}^{*}$ (the figure above suggests $\bar{y}^{*}=10$ would be reasonable) this integral may approximately be split into two parts:

$$
\dot{n} \approx c^{*} \mathcal{D} \int_{0}^{\bar{y}^{*}} \bar{c}(\bar{y}) \mathrm{d} \bar{y}+c^{*} \mathcal{D} \int_{\bar{y}^{*}}^{\infty} \frac{2}{\pi} \frac{1}{\bar{y}} \mathrm{~d} \bar{y}
$$

The 2nd integral in the above expression fails to converge. This failure of convergence is caused by the fact that we are ignoring all length scales within the system except for $L_{s}$. In the actual system, the bulk reaction will cause $c \rightarrow 0$ exponentially quickly, with this exponential decay occuring over length scale $y \sim L_{b} \gg L_{s}$. This may be seen in the figure below, which shows numerically calculated concentration profiles along the cathode surface. As predicted in Eq. (92), $\bar{c}$ approaches $2 / \pi \bar{y}$ at large $\bar{y}$. However, when $\bar{y} / \lambda \sim 1$ (i.e. $\left.y \sim L_{b}\right)$ the profiles deviate and exponentially decay towards 0 .

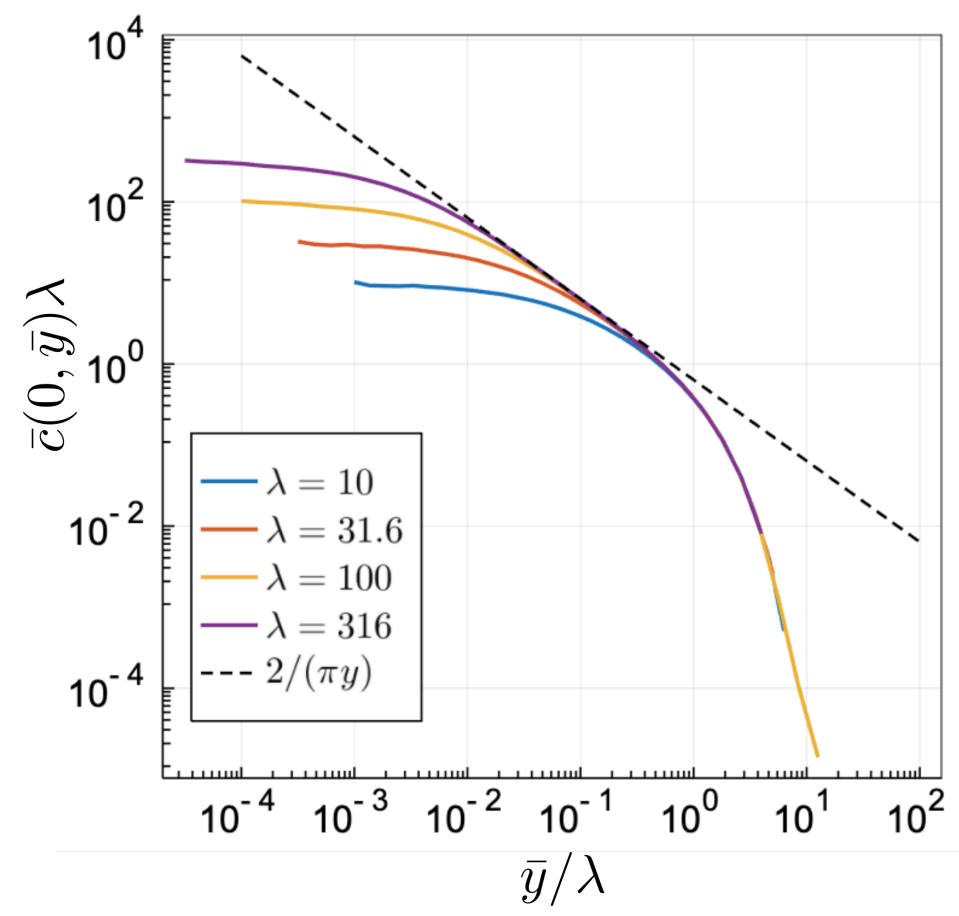

Figure S6: Dimensionless concentration profiles along catalyst surface.

Hence to a first approximation the second integral above may be be cut-off 
at values of $y \geq L_{b}$, which corresponds to $\bar{y} \sim L_{b} / L_{s}=\lambda$ :

$$
\begin{aligned}
\dot{n} & \approx c^{*} \mathcal{D} \int_{0}^{\bar{y}^{*}} \bar{c}(\bar{y}) \mathrm{d} \bar{y}+c^{*} \mathcal{D} \int_{\bar{y}^{*}}^{\lambda} \frac{2}{\pi} \frac{1}{\bar{y}} \mathrm{~d} \bar{y} \\
& =c^{*} \mathcal{D} \int_{0}^{\bar{y}^{*}} \bar{c}(\bar{y}) \mathrm{d} \bar{y}+c^{*} \mathcal{D} \frac{2}{\pi}\left(\log \lambda-\log \bar{y}^{*}\right)
\end{aligned}
$$

Choosing $\bar{y}^{*}=10$ and numerically evaluating the first integral via Eq. (91), this reduces to

$$
\frac{\dot{n}}{c^{*} \mathcal{D}}=0.374+\frac{2}{\pi} \log \lambda
$$

As may be seen in the figure below, this asymptotic expression is in excellent agreement with analytical solution at large values of $\lambda$. This scaling law may be considered a general property of mass-transfer limited behaviour within TPRs.

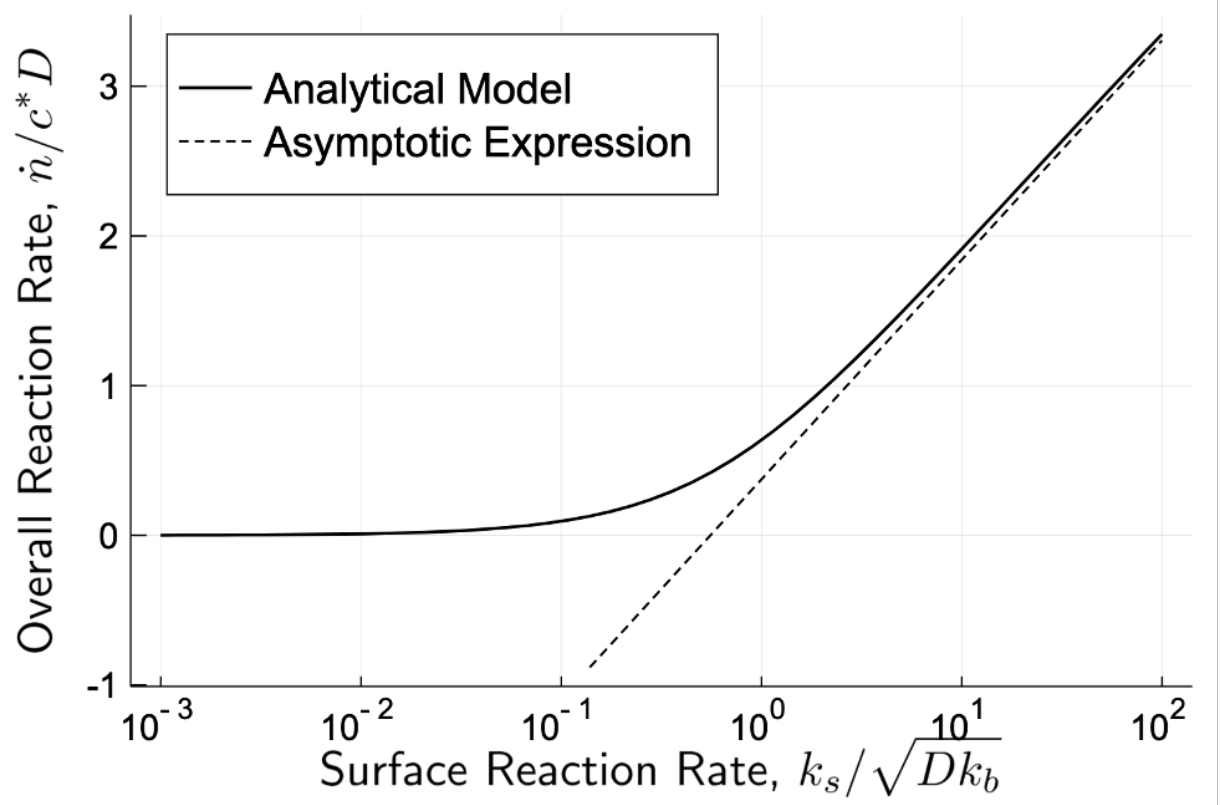

Figure S7: Overall reaction rate, predicted by complete analytical model and asymptotic expression for large $\lambda=k_{s} / \sqrt{D k_{b}}$. 


\section{Section S6: Nondimensionalization of Resolved $\mathrm{CO}_{2}$ Pore Model}

The reaction-diffusion pore model is defined by the following set of PDE's, which are solved on a semi-infinite pore (see Figure S3):

$$
\begin{gathered}
\nabla^{2} c=k_{b} c \\
\left.c\right|_{z=0}=c_{0} \\
-\left.D \frac{\partial c}{\partial r}\right|_{r=R}=k_{S} c \\
\left.\frac{\partial c}{\partial z}\right|_{z \rightarrow \infty}=0
\end{gathered}
$$

We define the following dimensionless variables:

$$
\bar{c} \equiv \frac{c}{c_{0}} ; \quad \bar{r}=\frac{r}{R / 2} ; \quad \bar{z}=\frac{z}{R / 2}
$$

A characteristic length-scale of $R / 2$ is used, as $\frac{R}{2}=\frac{1}{a}$ in a cylindrical pore. In dimensionless form the above PDE's reduce to:

$$
\begin{gathered}
\bar{\nabla}^{2} \bar{c}=\mathrm{Da}_{\mathrm{b}}^{2} \bar{c} \\
\left.\bar{c}\right|_{\bar{z}=0}=1 \\
\left.\frac{\partial \bar{c}}{\partial \bar{r}}\right|_{\bar{r}=2}=\mathrm{Da}_{\mathrm{s}} c \\
\left.\frac{\partial \bar{c}}{\partial \bar{z}}\right|_{\bar{z} \rightarrow \infty}=0
\end{gathered}
$$

Where $\mathrm{Da}_{\mathrm{s}}$ and $\mathrm{Da}_{\mathrm{b}}$ are Damköhler numbers for the surface and bulk reactions, respectively, and are defined as,

$$
\mathrm{Da}_{\mathrm{b}} \equiv \frac{R}{2} \sqrt{\frac{k_{b}}{D}} ; \quad \mathrm{Da}_{\mathrm{s}} \equiv \frac{R}{2} \frac{k_{s}}{D}
$$

Hence, $\mathrm{Da}_{\mathrm{s}}$ and $\mathrm{Da}_{\mathrm{b}}$ fully specify the dimensionless form of the resolved $\mathrm{CO}_{2}$ pore model. 


\section{Section S7: Comparison Between Resolved $\mathrm{CO}_{2}$ Pore Model \& Multiphysics Model}

In the figure below, the multiphysics pore model is systematically compared with the resolved $\mathrm{CO}_{2}$ pore model. The following conditions were considered:

- Pore Radius: 2 microns; Catalyst Layer $\mathrm{pH} \sim 14$

- Pore Radius: 2 microns; Catalyst Layer $\mathrm{pH} \sim 15$

- Pore Radius: 0.2 microns; Catalyst Layer $\mathrm{pH} \sim 14$

For each case, the cathode potential was varied, causing the $\mathrm{CO}_{2} \mathrm{R}$ current density to vary by several orders of magnitude, and each system to transition from $1 \mathrm{D}$ bulk or surface reaction control to TPB control.
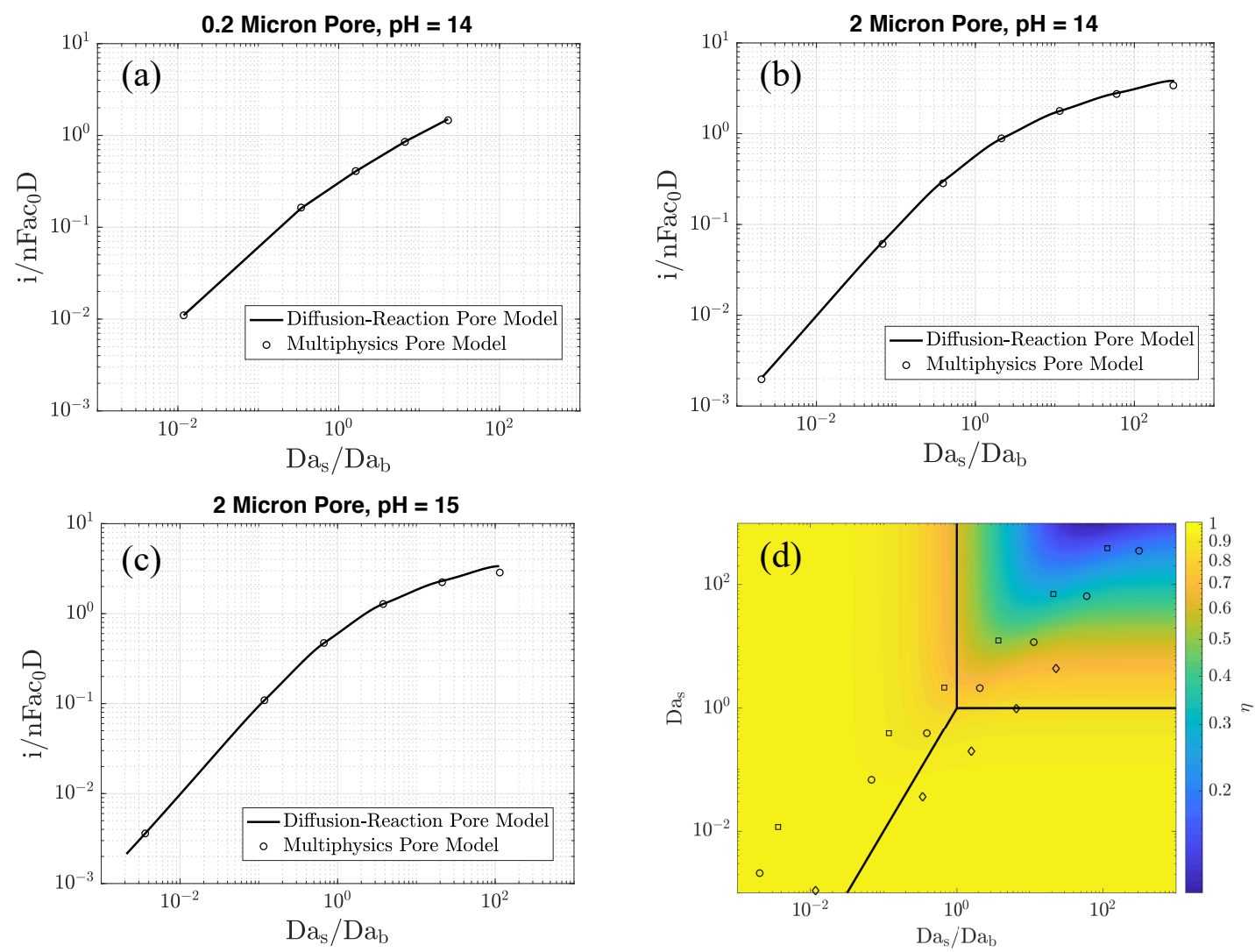

Figure S8: Comparison between Multiphysics Model and Resolved $\mathrm{CO}_{2}$ Pore Model for (a) Pore Radius: 0.2 microns; Catalyst Layer pH 14 (b) Pore Radius: 2 microns; Catalyst Layer pH 14 (c) Pore Radius: 2 microns; Catalyst Layer $p H \sim 15$. (d) Location of simulations in (a) $-(c)$ on operational phase diagram. Circles: Pore Radius: 2 microns; Catalyst Layer pH $\sim 14$.

Diamonds: Pore Radius: 0.2 microns; Catalyst Layer pH 14. Squares: Pore Radius: 2 microns; Catalyst Layer $\mathrm{pH} \sim 15$. 
For all simulations, the relative error was less than $20 \%$, and for all simulations with $D a_{s} / D a_{b}<$ 100 (i.e. outside of extremely strong TPR control), the relative error was less than 5\%, and the average relative error was $1.6 \%$. The excellent agreement between the two models is unsurprising, as variations in overpotential and $\mathrm{pH}$ within several microns of the TPR are likely to be small, especially outside of the strongly TPR controlled regime. 


\section{Section S8: Use of Figure 4}

In the table below, the calculation of $\mathrm{Da}_{\mathrm{b}}$ and $i_{\mathrm{CO}_{2} R} / n F a c_{0} D$ for 'Point 1' in Figure $4 \mathrm{a}$ of the main paper is outlined. We first list values for physical parameters, before evaluating dimensionless groups. Notes are provided where physical parameters are uncertain. Methods for estimating the the catalyst-layer $\mathrm{pH}$ are then discussed.

Table 1: Parameters for 'Point 1' in Figure 4a

\begin{tabular}{|c|c|c|c|c|}
\hline Parameter & Description & Value & Source & Notes \\
\hline$n$ & $\begin{array}{l}\text { moles electrons per } \\
\text { moles } \mathrm{CO}_{2}\end{array}$ & 2 & $\mathrm{~N} / \mathrm{A}$ & $\begin{array}{c}\text { Moles of electrons per mole } \mathrm{CO}_{2} \\
\text { converted to } \mathrm{CO}\end{array}$ \\
\hline$F$ & Faraday's Constant & $96485 \mathrm{C} \mathrm{mol}^{-1}$ & $\begin{array}{l}\text { Newman et al. } \\
2012\end{array}$ & 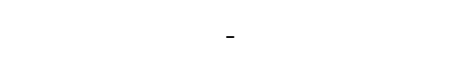 \\
\hline$a$ & $\begin{array}{l}\text { Specific catalyst } \\
\text { surface area }\end{array}$ & $1 \times 10^{7} \mathrm{~m}^{2} \mathrm{~m}^{-3}$ & $\begin{array}{c}\text { Based on } \\
R_{p}=200 \mathrm{~nm}\end{array}$ & $\begin{array}{l}\text { May be estimated via } a \approx 2 / R_{p} \text { or } \\
a \approx 2 \varepsilon / R_{p}, \text { were } R_{p} \text { is the pore } \\
\text { radius and } \varepsilon \text { is catalyst liquid } \\
\text { fraction. May also be estimated } \\
\text { via electrochemical surface area } \\
\text { (ECSA) measurement. }\end{array}$ \\
\hline$c_{0}$ & $\begin{array}{l}\text { Equilibrium } \mathrm{CO}_{2} \\
\text { Concentration }\end{array}$ & $25 \mathrm{~mol} \mathrm{~m}^{-3}$ & $\begin{array}{l}\text { Versteeg and } \\
\text { Van Swaaij } \\
\quad(1988)\end{array}$ & $\begin{array}{l}\text { Strong temperature and } \\
\text { composition dependence. See, for } \\
\text { instance, Weisenberger and } \\
\text { Schumpe (1996) }\end{array}$ \\
\hline$D$ & $\mathrm{CO}_{2}$ Diffusivity & $1.91 \times 10^{-9} \mathrm{~m}^{2} \mathrm{~s}^{-1}$ & $\begin{array}{l}\text { Weng et al. } \\
2018\end{array}$ & $\begin{array}{l}\text { Weak temperature and } \\
\text { composition dependence. See, for } \\
\text { instance, Versteeg and Van Swaaij } \\
\text { (1988) }\end{array}$ \\
\hline$i_{\mathrm{CO}_{2} \mathrm{R}}$ & $\begin{array}{l}\text { Geometric } \mathrm{CO}_{2} \mathrm{R} \\
\text { Current Density }\end{array}$ & $400 \mathrm{~mA} \mathrm{~cm}^{-2}$ & Assumed & Specified by user. \\
\hline$c_{\mathrm{OH}^{-}}$ & Catalyst-layer pH & $1 \mathrm{M}$ & Assumed & $\begin{array}{l}\text { Not equal to bulk electrolyte } \mathrm{pH} \text {. } \\
\text { Estimation methods discussed } \\
\text { below. }\end{array}$ \\
\hline$k_{2}$ & $\begin{array}{l}\text { Second-order Rate } \\
\text { constant for Rxn (1) } \\
\text { in paper. }\end{array}$ & $21901 / \mathrm{Ms}$ & $\begin{array}{l}\text { Weng et al. } \\
2018\end{array}$ & $\begin{array}{l}\text { Significant uncertainty in } \\
\text { literature. c.f. Table } 3 \text { of Borhani } \\
\text { et al (2015). }\end{array}$ \\
\hline$\frac{{ }^{i} \mathrm{CO}_{2} R}{n F a c_{0} D}$ & $\begin{array}{l}\text { Dimensionless } \mathrm{CO}_{2} \mathrm{R} \\
\text { current density. }\end{array}$ & 0.043 & Calculated & $\begin{array}{c}\text { Calculated from parameters } \\
\text { above. }\end{array}$ \\
\hline $\begin{array}{l}D a_{b}= \\
\frac{1}{a} \sqrt{\frac{k_{2} c}{\mathrm{OH}^{-}}}\end{array}$ & $\begin{array}{l}\text { Bulk Damköhler } \\
\text { Number }\end{array}$ & 0.11 & Calculated & $\begin{array}{c}\text { Calculated from parameters } \\
\text { above. }\end{array}$ \\
\hline
\end{tabular}

\section{Estimating Catalyst-Layer pH}

The governing mass-transport mechanism within the catalyst layer is a strong function of the local $\mathrm{pH}$ within the catalyst layer. This $\mathrm{pH}$ is influenced by 
three physical processes: the consumption of $\mathrm{OH}^{-}$by $\mathrm{CO}_{2}$, the production of $\mathrm{OH}^{-}$at the catalyst surface, and the diffusion and migration (possibly accompanied by chemical reaction) of $\mathrm{OH}^{-}$between the catalyst surface and the bulk electrolyte (Burdyny and Smith, 2019).

As a first approximation, Figure 3d of Burdyny and Smith (2019) and Figure 2 of Lamaison et al (2021) (with the accompanying equations described in the ESI) may be used to estimate the catalyst-layer $\mathrm{pH}$. The former assumes a fixed boundary layer thickness of $200 \mu \mathrm{m}$, while the latter allows the boundary layer thickness to be varied.

For a more rigorous calculation, a 1D boundary layer model may be constructed (see, e.g., Burdyny and Smith (2019).) These 1D models require a boundary layer thickness. This is typically of the order of $100 \mu \mathrm{m}$. It may be estimated more rigorously from the following equation if a Sherwood number may be estimated for the particular electrolyte flow configuration (Lamaison et al. 2021; Bird et al. 2002):

$$
\delta=\frac{L}{\mathrm{Sh}}=\frac{D}{k_{L}}
$$

where $\delta$ is the boundary layer thickness, Sh is the average Sherwood number across the electrode, $L$ is the characteristic length used in the calculation of the Sherwood number (e.g. electrode length), $D$ is the $\mathrm{OH}^{-}$diffusivity, and $k_{L}$ is the $\mathrm{OH}^{-}$mass transfer coefficient. Correlations for the Sherwood number in several common configurations are provided by Newman (2012).

$1 \mathrm{D}$ models fail to account for $2 \mathrm{D}$ or $3 \mathrm{D}$ flow effects. A more rigorous means of estimating the catalyst-layer $\mathrm{pH}$ involves the construction of flow simulations, such as were considered by Kas et al. (2021). 


\section{References}

- Bird, R. B., W. E. Stewart, and E. N. Lightfoot. Transport Phenomena. $2^{\text {nd }}$ Ed. John Wiley \& Sons." (2002).

- Borhani, Tohid Nejad Ghaffar, et al. "CO2 capture with potassium carbonate solutions: A state-of-the-art review." International journal of greenhouse gas control 41 (2015): 142-162.

- Burdyny, Thomas, and Wilson A. Smith. "CO 2 reduction on gas-diffusion electrodes and why catalytic performance must be assessed at commercially-relevant conditions." Energy \& Environmental Science 12.5 (2019): 1442-1453.

- Cussler, Edward Lansing. Diffusion: mass transfer in fluid systems. Cambridge university press, 2009.

- Danckwerts, Peter Victor. Gas-liquid reactions. (1970). McGraw-Hill

- Fukunaka, Y., et al. "Nonuniformity of $\mathrm{NaOH}$ concentration and effective bubble diameter in CO 2 injection into aqueous NaOH solution." Metallurgical Transactions B 20.1 (1989): 5-12.

- Haberman, Richard. Applied partial differential equations. 2003.

- Kas, Recep, et al. "Along the Channel Gradients Impact on the Spatioactivity of Gas Diffusion Electrodes at High Conversions during CO2 Electroreduction." ACS Sustainable Chemistry \& Engineering 9.3 (2021): 1286-1296.

- Lamaison, S., Wakerley, D., Kracke, F., Moore, T., Zhou, L., Lee, D., Wang, L., Hubert, M., Acosta, J., Gregoire, J., Beck, V., Spormann, A., Fontecave, M., Hahn, C., and Jaramillo, T. 2021. Outcompeting precious metals through catalyst layer engineering of a $\mathrm{Zn}-\mathrm{Ag}$ GDE for $\mathrm{CO}$ production amenable to microbial conversion to acetate and ethanol. Advanced Materials. Accepted for Publication.

- Lv, Jing-Jing, et al. "A highly porous copper electrocatalyst for carbon dioxide reduction." Advanced Materials 30.49 (2018): 1803111.

- Newman, John, and Karen E. Thomas-Alyea. Electrochemical systems. John Wiley \& Sons, 2012.

- O’Hayre, Ryan, David M. Barnett, and Fritz B. Prinz. "The triple phase boundary: a mathematical model and experimental investigations for fuel cells." Journal of the Electrochemical Society 152.2 (2005): A439.

- Tjaden, Bernhard, et al. "On the origin and application of the Bruggeman correlation for analysing transport phenomena in electrochemical systems." Current Opinion in Chemical Engineering 12 (2016): 44-51.

- Versteeg, Geert F., and Wim PM Van Swaaij. "Solubility and diffusivity of acid gases (carbon dioxide, nitrous oxide) in aqueous alkanolamine solutions." Journal of Chemical \& Engineering Data 33.1 (1988): 29-34.

- Weisenberger, S., and dan A. Schumpe. "Estimation of gas solubilities in salt solutions at temperatures from $273 \mathrm{~K}$ to $363 \mathrm{~K} . "$ AIChE Journal 42.1 (1996): 298-300.

- Weng, Lien-Chun, Alexis T. Bell, and Adam Z. Weber. "Modeling gas-diffusion electrodes for CO2 reduction." Physical Chemistry Chemical Physics 20.25 (2018): 16973-16984.

- Weng, Lien-Chun, Alexis T. Bell, and Adam Z. Weber. "Towards membrane-electrode assembly systems for CO 2 reduction: a modeling study." Energy \& Environmental Science 12.6 (2019): 1950-1968. 
- Wu, Jingjie, et al. "Electrochemical reduction of carbon dioxide: IV dependence of the Faradaic efficiency and current density on the microstructure and thickness of tin electrode." Journal of Power Sources 258 (2014): 189-194. 